\title{
Anger and the speed of full-body approach and avoidance reactions
}

\author{
Iddo Mayan and Nachshon Meiran* \\ Department of Psychology, Ben-Gurion University of the Negev, Beer-Sheva, Israel
}

\section{Edited by:}

Marco Tamietto, Tilburg University,

Netherlands

Reviewed by:

Saskia Van Dantzig, Leiden University,

Netherlands

Jan Van den Stock, Tillburg University,

Netherlands

*Correspondence:

Nachshon Meiran, Department of

Psychology, Ben-Gurion University of

the Negev, Beer-Sheva 84105, Israel.

e-mail:nmeiran@bgu.ac.il
The notion that anger is linked to approach motivation received support from behavioral studies, which measured various motor responses to angering stimuli. However, none of these studies examined full-body motions which characterize many if not most everyday instances of anger. The authors incorporate a novel behavioral motor task that tests motivational direction by measuring the reaction times (RTs) of stepping forward and backward in response to the words "toward" and "away." The results show that, relative to anxiety and control conditions, anger induction resulted in a steeper approach-avoidance RT gradient which was shifted in favor of approach.

Keywords: anger, action tendencies, reaction time, full-body movement

\section{INTRODUCTION}

Different emotional processes involve activation of either left or right frontal areas of the brain (see Coan and Allen, 2004; HarmonJones, 2007). Relatively greater left frontal activity is typically associated with greater positive affect (e.g., happiness, joy), whereas relatively greater right frontal activity is associated with greater negative affect (e.g., sadness, disgust, fear; see Harmon-Jones, 2007). However, Harmon-Jones's work (see Harmon-Jones and Allen, 1998; Harmon-Jones, 2007; Carver and Harmon-Jones, 2009) suggests that the hemispherical differences are not an indication of affective valence (feeling a positive or negative emotion), but of motivational tendencies of approach or withdrawal (toward or away from the stimulus). Anger constitutes a critical case that can help to differentiate between these two interpretations of hemispheric asymmetry. This is because anger is frequently experienced as a negatively valenced and unpleasant emotion, but is typically associated in the literature with approach behavior, i.e., approaching with the intent of removing the stimulus (e.g., Harmon-Jones, 2003; Carver, 2004; Carver and Harmon-Jones, 2009), typically associated with arousal and an urge to attack or injure (e.g., Hoeksma et al., 2007). Anger is seen as an event triggered by goal blockage (Lazarus, 1966), or a "challenge of what ought to happen" (Frijda, 1986). It arises when individuals feel that they are kept by another's improper action from attaining a goal, yet feel capable of altering the situation (Frijda, 1986). In other words, anger is evoked when our goal-directed action is disrupted. Accordingly, key behavioral aspects of anger are aggression (e.g., Kassinove, 1995; Berkowitz and Harmon-Jones, 2004) and confrontation (Parrott, 2001), and individuals may become angry when expecting situations involving these actions (Tamir et al., 2008).

Neurological investigations also support the notion that anger is an approach related affect. Left prefrontal activity increases following exposure to positive incentive stimuli (see Coan and Allen, 2004), but it also increases following angering interpersonal provocations (Harmon-Jones and Sigelman, 2001; Harmon-Jones et al., 2004, 2006).
Many studies sought to associate affects with approach or avoidance behavior. In an early study participants were required to push cards with words either toward themselves or away from themselves (Solarz, 1960). Chen and Bargh (1999) used lever pulling and pushing. Other studies used arm flexion and extension movements (e.g., Neumann and Strack, 2000; Cretenet and Dru, 2004; Centerbar and Clore, 2006). In a study by Wentura et al. (2000), participants made lexical decisions with adjectives as word stimuli. Some adjectives described things that are good or bad to a person if someone else possesses them such as "fair" and "aggressive," respectively. The results show relatively quick button presses (representing approach) in response to positive adjectives and relatively quick button releases (representing avoidance) in response to negative adjectives. In order to strengthen the association between pressing/releasing and approach/avoidance, the respective responses were accompanied with increases and decreases in stimulus size. Additional studies measured responses that decrease or increase the distance between the participant and the stimulus as a measure of approach and avoidance (e.g., Markman and Brendl, 2005; Lavender and Hommel, 2007; van Dantzig et al., 2008).

A limitation of all the above studies is that the participants operated levers or buttons that caused a clear change in the stimulus or changed the participant's perceived location, but did not consist of an actual movement of the full body toward or away from the stimulus to guarantee that the participant actually had approach or avoidance motivation. A promising new approach was introduced by Chajut et al. (2010) who introduced a reaction time (RT) task involving the full-body motion of walking forward and backward on a "dance mat," as a response to valenced stimulus words presented on a computer screen.

Several studies examined the association between anger and approach motor behavior. Lewis et al. (1990) found that infants who showed more angry expressions in a learning task, showed more active hand pulling movement, which might signify aggression and approach motivation. Buss and Goldsmith (1998) additionally found the use of approach motor behavior reduced the observable 
intensity of anger. Newhagen (1998) presented participants with television news' images that induced anger, fear or disgust. The participants were instructed to rate the image by moving a computer paddle toward or away from the on-screen image, according to whether they wanted to get nearer or move away from the people in the image. The results indicated that anger images received the rating closest to the screen. Marsh et al. (2005) showed that angry faces facilitated arm extension and that fear faces facilitated arm flexion. Adams et al. (2006) introduced a behavioral task that gaged the speed with which angry and fearful faces (which moved toward or away from a participant's gaze) were accurately detected. It was found that participants were quicker to correctly detect approaching anger faces (i.e., faces that moved in the direction of their own gaze) but not fearful faces. Wilkowski and Meier (2010) used a distance regulation method in order to measure motor behavior as a response to angry or neutral facial expressions. They instructed participants to move an on-screen representation of themselves (the word "ME," presented at the center of the screen) toward or away from the presented expression, and found that forward movements were initiated faster toward angry expressions.

The studies reviewed above, while providing evidence for approach intent are limited in the same respect: the behavioral tasks did not require an actual full-body movement indicative of approach or avoidance during the induced anger, thus not fully representing approach or avoidance motivation. Our main goal was to link anger to motivational direction, using simple forward and backward walking movement RT time task, during induced anger. In our study, we asked participants to stand on a "dance mat" placed in front of the computer monitor and asked them to simply step forward in response to the stimulus word "toward" and step backward in response to the stimulus word "away" while we measured RT (similar to the procedures used in Chajut et al., 2010). The participants were asked to react as quickly as possible and were led to believe that they were competing against an opponent (similar to procedures used in Anderson and Bushman, 1997). To induce anger, we used several established manipulations without any attempt to assess their specific contribution. Rather, we were interested in ensuring as much as possible that a genuine feeling of anger would be evoked. The manipulations involved frustration (Berkowitz, 1989), unfairness (Weiss et al., 1999), unpleasant conditions (Berkowitz, 1990), and interpersonal harassment (Lobbestael et al., 2007; Quartana and Burns, 2007). In addition, we primed the participants for anger by asking them to recall in detail an angry experience (Rusting and NolenHoeksema, 1998) and had them listen to a piece of grossly atonal piece of music that was previously judged to generate unrest. Specifically, the critical steps in our procedure were the following. The participants first listened to a piece of music designed to induce a given emotion, then completed an event recall procedure (in the anger and control groups). The next stage involved the RT testing in which the participants were led to believe that they compete against a student from another university. In the anger group, the rules of this "competition" were that the winner induces unpleasant sound in the ear of the opponent and can control the level of this noise. In addition to these critical steps, the participants filled some questionnaires and mood checks as described below.
Aside from running a control group we also included an anxiety group. We chose to induce anxiety because this emotion, like anger, is a negative affect involving physiological arousal, but unlike anger this emotion is not believed to be associated with increased approach motivation. Including this group made it possible for us to rule out the (not very likely) interpretation that the increased approach tendency was a reflection of negative emotion in general, as opposed to anger in particular.

To validate the mood induction we used self reports as mood checks. We also made an effort to obtain a behaviorally based measure of aggression, which was the mean sound level that the participant chose for her opponent.

Our primary dependent measure was the RT gradient of approach and avoidance $\left(\mathrm{RT}_{\text {avoid }}-\mathrm{RT}_{\text {approach }}\right)$. This gradient reflects the relative balance between approach and avoidance tendencies and higher values indicate a bias in favor of approach. We hypothesized that relative to non-angered participants and conditions in which anger was not yet induced, the gradient would shift in favor of approach in the experimental blocks in which anger was successfully induced.

\section{MATERIALS AND METHODS PARTICIPANTS}

Participants were 30 healthy psychology undergraduate students (all female) who were recruited at Ben-Gurion University of the Negev, mean age 23.87 years ( $S D=1.59$ years). They participated in exchange for course credit. We used females only in order to make the groups homogenous and thus increase the statistical power. We chose to run females and not males because most of the students in the department are women. The participants were randomly assigned to one of the three groups, 10 in each group: anger induction, anxiety induction, and neutral affect (control).

\section{MATERIALS}

\section{Consent forms}

The consent forms stated that the experiment tests the effect of emotional experience on movement speed, which is checked in a movement speed competition, performed online against a student from Tel Aviv University.

\section{Mood checks}

Participants rated their emotions four times during the experiment at the time points described below. Mood checks 1 and 4 contained 10 emotions, presented in random order: happiness, anger, sadness, humiliation, anxiety, calmness, fear, irritation, hostility, and general positive mood. Mood checks 2 and 3 involved four emotions: anger, anxiety, irritation, and general positive mood. Specifically, participants were asked to rate the intensity of each emotion (e.g., "How happy are you right now?" "How angry are you right now?"). Emotion intensities were displayed as values from 1 to 9 , which participants could click on and a verbal description was associated with each number (e.g., $1=$ not at all, $9=$ very much so).

\section{Dance mat and RT measurement}

In order to assess the forward and backward RTs, participants were placed on an electronic dance mat (iDDR - "Israeli Dance Dance Revolution"@, http://www.iddr.co.il, see Figure 1). The mat was 


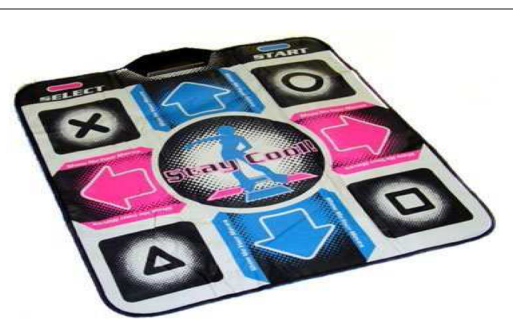

FIGURE 1 |The Dance Dance Revolution Mat (http://www.iddr.co.il) used for reaction time measurement.

$80 \mathrm{~cm} \times 80 \mathrm{~cm}$. The participants stood on the center square and the distance from this square to the up and down keys which were used was $28 \mathrm{~cm}$. The dance mat emulates the arrow key presses which were recorded by the desktop computer that was attached to it and to a $17^{\prime \prime}$ monitor.

\section{State-Trait Anger Expression Inventory-2}

The State-Trait Anger Expression Inventory-2 (STAXI-2) is a 57-item self report scale that was developed by Spielberger (1996) to quantify the experience, expression, and control of anger, both at the state and trait levels. The STAXI-2 has a 15-item scale that measures state-anger. Other items measure overall trait anger, trait anger expression, and the control of anger. The STAXI-2 has been found to be a valid and reliable measure of the constructs it was intended to represent (Spielberger, 1996).

\section{Mood restoration}

A short funny story ("Shriki” by Keret, 2002) was used in order to restore neutral or positive mood in the participants. The story was found to neutralize negative mood in a pilot study.

\section{PROCEDURE}

\section{Introductory phase}

Testing was done individually. After greeting the participants, the experimenter asked them to fill out an informed consent form and stressed the importance of starting the experiment on time, since the experiment is performed online and consists of a competition against a student from another university in Israel (Tel Aviv University) and that it is crucial to start together.

After filling out the consent forms, participants entered the testing room and were told to take off their shoes for the use of the dance mat, which was currently put aside. Participants were told to sit in front of the computer monitor for the initial phase of the experiment, which involved an on-screen introduction, demographics questions and mood check 1. Afterward, a practice phase of the approach/avoidance task ensued to ensure understanding of the task and the use of the mat.

\section{Practice phase}

The practice phase consisted of 12 trials ( 6 for approach, 6 for avoidance). The participants were reminded that speed is important, particularly in the context of the "contest" with the other student. They were placed about $1 \mathrm{~m}$ from the computer monitor. Their task was to step forward on the "up" key of the "dance mat" or backward on the "down" key of the dance mat in response to the on-screen Hebrew words for "toward" and "away." Although we did not instruct the participants which foot to use, the vast majority used the right foot for both responses while standing on their left foot. Following a presentation of a " + " sign in the middle of the screen for $1000 \mathrm{~ms}$, these words were presented in the middle of the screen in 28 point size Times New Roman font, and disappeared when the correct arrow on the mat was pressed with the participant's foot. The participants were told to fully press the forward/backward arrows according to the word that appeared, and then return to the "home" space.

\section{Music phase}

The participants were instructed to put on headphones and listen to a 6-min musical piece. The anger group listened to "Stockhausen Recomposed" by Jim Jandt, a contemporary and grossly dissonant piece, found in a pilot study to increase reports of unrest and irritability. The anxiety group listened to "Halloween" by John Carpenter, which was found to induce anxiety in previous research (Moritz et al., 2009). The control group listened to "Spacewalker" by Depeche Mode, an instrumental piece found in a pilot study not to induce any particular emotion.

\section{Event recall}

Participants were instructed to put on headphones and listen to instructions detailing the recollection of an emotional experience. The instructions directed the participants to vividly recollect an event and write it down. The anger group received instructions for recollecting an angering and humiliating experience:

\begin{abstract}
"Take a few moments to think about times that you were angry about a friend, family member or any other person because you felt he or she humiliated you or did you wrong, and you wanted to get back at them for it and make that person hurt."
\end{abstract}

The control group was asked to recall a neutral experience:

\begin{abstract}
"Take a few moments to think of a memory in which you were in contact with another person, and that person did not make you feel any emotion. It could be a friend, a family member, or any other person."
\end{abstract}

\section{Anxiety induction}

For the anxiety group, a visible video camera was put inside the room. The participants were informed that the camera would be turned on during the competition phase and that the recorded video would be presented to raters afterward so that the participants' stress level could be assessed by watching their facial expressions during the competition.

\section{Competition phase}

Following the event recall phase, the participants were told that the competition was about to begin. The experimenter entered the other room in order to "check" if the other student was prepared, and told the participant that the competition would now begin. The competition consisted of three blocks of trials.

For the anger group, the participants were instructed to put on the headphones. The experimenter explained: "During the competition, each time a competitor loses (is slower than the opponent), 
he/she hears a noise. Before each trial, both sides are prompted to select the noise volume to be presented to their opponent, if he/ she loses. The noise level is on a scale of $1-100$."

The computer volume was set so the noise of the highest volume heard by the participants (computer volume at $25 \%$ for all participants) would be clearly heard, but not harmful. The number of trials the participants lost and the noise levels the participants heard were in fact predetermined because the opponent was fictitious.

The first block consisted of 32 trials (16 approach trials and 16 avoidance trials), $50 \%$ of which the participants won, and $50 \%$ of which they lost. The second and third rounds consisted of 44 trials each ( 22 of approach and 22 of avoidance) in which the participants in the anger group "lost" the competition in $80 \%$ of the trials while in the other groups they lost $50 \%$ of the trials.

In the anger group, when losing the competition in a given trial, the participants heard a noise (volume levels ranged between 30 and $60 \%$ of the maximum in block 1 and between 60 and 90\% in blocks 2 and 3) over the headphones. As mentioned above, the levels of noises administered by the anger group participants were recorded.

In each trial, following the approach/avoidance task described above (in the practice phase), a message saying "checking reaction times..." appeared for $3000 \mathrm{~ms}$, followed by a message relating to the participant whether she/he won or lost. The message for a "win" trial was, "You won! Your opponent will now hear a noise in volume $\mathrm{X}$," with X being the volume the participant chose before the trial. The message for a "lose" trial was, "You lost. You will now hear a noise in volume Y," with Y being the volume randomly chosen for the participant to hear in that trial (see above). The noise began $500 \mathrm{~ms}$ after the message appeared, and was superimposed on the message screen. The noise was a "buzz" sound judged as being irritating by participants in a pilot study. The buzz sounded three times, for 1000 ms each. Afterward, a “+” sign appeared again in the middle of the screen for $1000 \mathrm{~ms}$, and the next trial ensued. RTs for the forward and backward movements on the dance mat were recorded as well as the accuracy of these responses.

After each competition block, the participants' mood was checked (mood checks 2, 3, and 4 after the first, second, and third blocks of the competition phase, respectively). At the end of the competition phase, participants completed the STAXI-2. Following the completion of the STAXI-2, participants the anger and anxiety groups were given the mood restoration. Finally, all the participants were debriefed and the rationale of the experiment was explained to them.

\section{RESULTS AND DISCUSSION MOOD CHECKS}

We ran a series of repeated measures two-way analyses of variance (ANOVAs) according to group and mood check point on each reported mood (Figures $\mathbf{2}-\mathbf{5}$ for the mean values). The twoway interaction was significant for anger, $F(6,81)=2.92, p<0.01$ and approached significance for anxiety, $F(6,81)=2.09, p=0.068$. Hostility was checked only in mood checks 1 and 4 . For this emotion, the interaction between group and mood check approached significance, $F(2,27)=3.06, p=0.067$. The results for irritation were non-significant. We also ran a series of planned contrasts comparing the reported emotions in a given time point to the same emotion as measured in baseline (mood check 1 ). These contrasts,
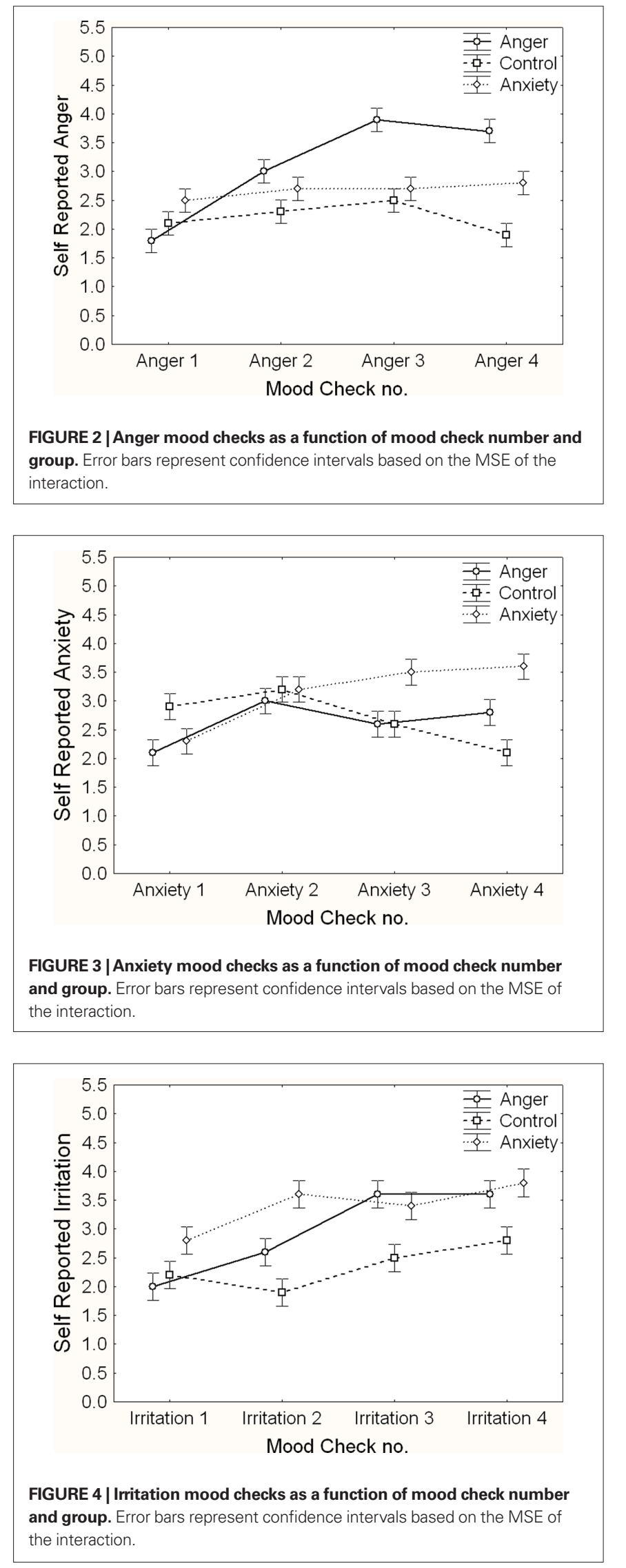
detailed in Table 1, reveal that, relative to baseline, participants in the anger group became more angry hostile and irritated, participants in the anxiety group became somewhat more anxious (the results were only close to significance), hostile and irritated and the results for the control group did change relative to baseline. Other effects were not significant.

\section{STATE-TRAIT ANGER EXPRESSION INVENTORY-2}

Our predictions regarding the participants' scores on the STAXI-2 questionnaire, filled out at the end of the experiment, were that the anger group would show more state-anger than others did, and that there would be no trait anger differences between groups, due to random selection. Contrary to expectation, the effect of emotion induction on state-anger scores was non-significant, $F(2,27)=0.88$, $p=0.43$. The mean state-anger score was only slightly higher for the anger group compared to the other groups $(M=18.6,17.4$, and 17.3 for anger, control, and anxiety, respectively). This lack of significance contrasts with the results of a pilot experiment, in which we found a significant difference between the anger and control groups. A possible explanation for this finding could be the longer duration of the competition phase in this experiment as compared with the pilot, such that more time elapsed over which the participants' anger could diminish. Note that this implies lesser differences in anger between the groups, which theoretically would make it more difficult to support our core prediction. The effect of emotion induction on trait anger scores was clearly non-significant, $F(2,27)=0.024, p=0.98$, thus validating the success of creating equivalent groups by the random assignment.

\section{NOISE LEVELS}

We predicted that the administered noise levels by the participants in the anger group (indicating anger-related aggression) would increase as the anger induction strengthened in blocks 2 and 3 of the competition phase. The effect of block on noise levels was non-significant, $F(2,18)=1.65, p=0.22$. However, the mean noise level was higher for blocks 2 and 3 compared to block $1(M=54.81$, 49.95 compared to $M=41.70$, respectively), which falls in line with our predictions. The explanation for the lack of significance is not necessarily a weak anger induction. Based on our debriefing we suggest another explanation for this null finding according to which the participants were careful not to increase the noise level, in order to avoid a similar reaction from their opponent.

\section{EFFECTS OF EMOTION ON APPROACH AND AVOIDANCE RTS}

We ran a mixed model three-way ANOVA on the RT results with group (anger/anxiety/control) as a between-subjects variable, and movement direction (approach vs. avoidance) and block as within-subjects variables. The three-way interaction was significant, $F(4,54)=3.78, p<0.01$ (Figure 6).

Because the approach-avoidance gradient serves as the indicator of approach tendencies, the simple two-way interaction between group and movement direction tests the difference between groups in their approach tendencies within a given block. An examination of this interaction in block 1 (in which the angry participants were not as angry as in blocks 2 and 3 ) was not significant, $F(2,27)=1.15$, $p=0.33$, as would be expected. In contrast, during blocks 2 and 3 , the parallel simple two-way interaction was significant, $F(2,27)=16.61$, $p<0.01$, revealing a distinct pattern of a shift toward approach in angry participants. A useful way to describe the triple interaction is based on the RT gradient $\left(\mathrm{RT}_{\text {avoidance }}-\mathrm{RT}_{\text {approach }}\right)$. The triple

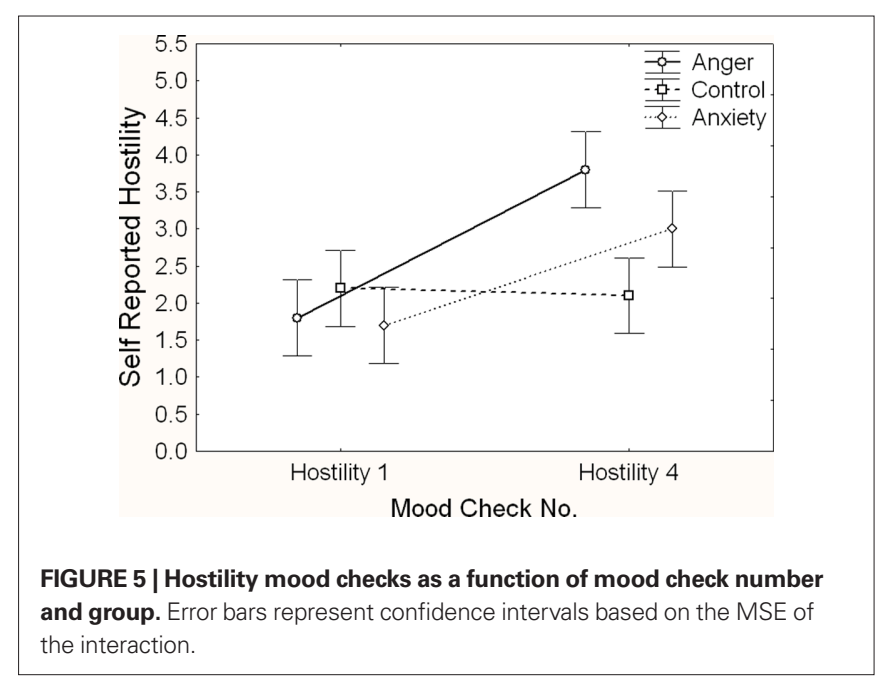

Table 1 | Planned contrasts for the mood checks*.

\begin{tabular}{|c|c|c|c|c|c|}
\hline $\begin{array}{l}\text { Emotion } \\
\text { rated }\end{array}$ & $\begin{array}{l}\text { Emotion } \\
\text { induced }\end{array}$ & $\begin{array}{l}\text { Mood points } \\
\text { contrasted }\end{array}$ & $F$ & MSE & $p$ \\
\hline Anger & Anger & 1 vs. 2 & 7.20 & 6.39 & $0.018^{*}$ \\
\hline Anger & Anger & 1 vs. 3 & 22.05 & 20.22 & $<0.001 *$ \\
\hline Anger & Anger & 1 vs. 4 & 18.05 & 29.90 & $<0.001 *$ \\
\hline Anger & Control & 1 vs. 2 & 0.20 & 0.18 & 0.677 \\
\hline Anger & Control & 1 vs. 3 & 0.80 & 0.73 & 0.399 \\
\hline Anger & Control & 1 vs. 4 & 0.20 & 0.33 & 0.570 \\
\hline Anger & Anxiety & 1 vs. 2 & 0.20 & 0.18 & 0.677 \\
\hline Anger & Anxiety & 1 vs. 3 & 0.20 & 0.18 & 0.672 \\
\hline Anger & Anxiety & 1 vs. 4 & 0.45 & 0.74 & 0.396 \\
\hline Anxiety & Anger & 1 vs. 2 & 4.05 & 3.13 & 0.088 \\
\hline Anxiety & Anger & 1 vs. 3 & 1.25 & 0.67 & 0.419 \\
\hline Anxiety & Anger & 1 vs. 4 & 2.45 & 2.01 & 0.168 \\
\hline Anxiety & Control & 1 vs. 2 & 0.45 & 0.35 & 0.560 \\
\hline Anxiety & Control & 1 vs. 3 & 0.45 & 0.24 & 0.626 \\
\hline Anxiety & Control & 1 vs. 4 & 3.20 & 2.63 & 0.117 \\
\hline Anxiety & Anxiety & 1 vs. 2 & 4.05 & 3.13 & 0.088 \\
\hline Anxiety & Anxiety & 1 vs. 3 & 7.20 & 3.88 & 0.059 \\
\hline Anxiety & Anxiety & 1 vs. 4 & 174.05 & 39.19 & $<0.001^{*}$ \\
\hline Hostility & Anger & 1 vs. 4 & 20.00 & 10.69 & $0.003^{*}$ \\
\hline Hostility & Control & 1 vs. 4 & 0.05 & 0.03 & 0.871 \\
\hline Hostility & Anxiety & 1 vs. 4 & 8.45 & 4.52 & $0.043^{*}$ \\
\hline Irritation & Anger & 1 vs. 2 & 1.80 & 1.38 & 0.249 \\
\hline Irritation & Anger & 1 vs. 3 & 12.80 & 7.95 & $0.009^{*}$ \\
\hline Irritation & Anger & 1 vs. 4 & 12.80 & 13.61 & $0.001 *$ \\
\hline Irritation & Control & 1 vs. 2 & 0.45 & 0.35 & 0.561 \\
\hline Irritation & Control & 1 vs. 3 & 0.28 & 1.61 & 0.601 \\
\hline Irritation & Control & 1 vs. 4 & 1.80 & 1.91 & 0.178 \\
\hline Irritation & Anxiety & 1 vs. 2 & 3.20 & 2.46 & 0.128 \\
\hline Irritation & Anxiety & 1 vs. 3 & 1.80 & 1.12 & 0.300 \\
\hline Irritation & Anxiety & 1 vs. 4 & 5.00 & 5.31 & $0.029 *$ \\
\hline
\end{tabular}

${ }^{*} p<0.05$. 


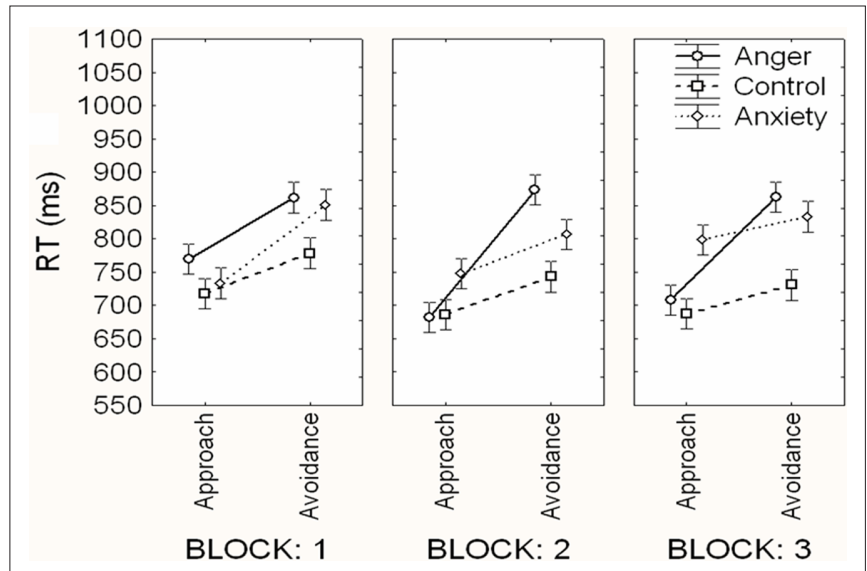

FIGURE 6 | Reaction time (RT in milliseconds) as a function of group, movement direction, and block. Error bars represent confidence intervals based on the MSE of the interaction.

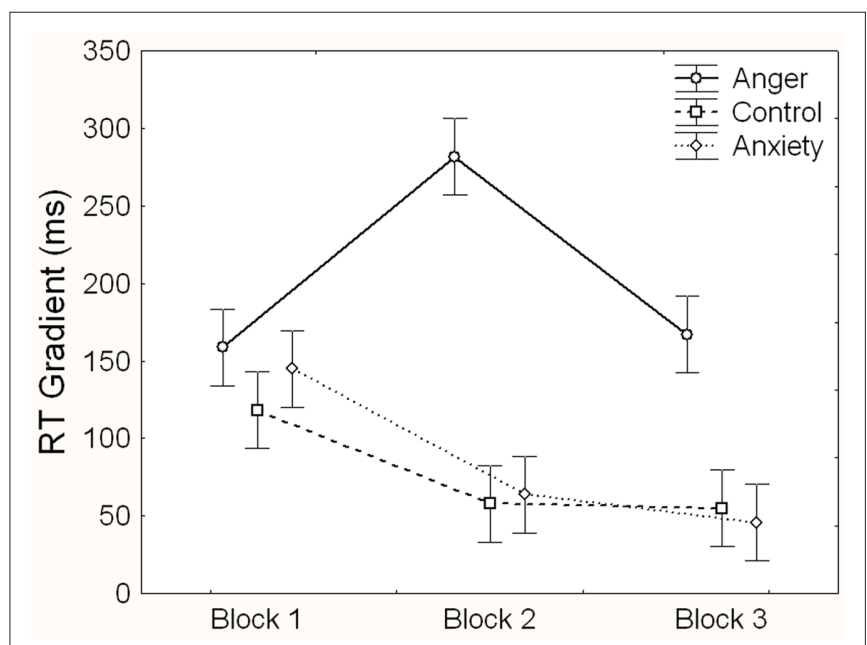

FIGURE 7 |The reaction time gradient $\left(R T_{\text {avoidance }}-\mathbf{R} T_{\text {approach }}\right.$ in milliseconds) as a function of group and block. Error bars represent confidence intervals computed based on the MSE of the triple interaction.

interaction implies that this RT gradient was significantly influenced by the interaction between group and block. As can be seen in Figure 7 , there was a shift in balance in favor of approach which appeared only in the anger group and only in the blocks in which this group lost most of the trials and reported increasing levels of anger.

\section{REFERENCES}

Adams, R.B.,Ambady,N.,Macrae,C.N., and Kleck, R. E. (2006). Emotional expressions forecast approach-avoidance behavior. Motiv. Emot. 30, 177-186.

Anderson, C.A., and Bushman, B. J. (1997). External validity of "trivial" experiments: the case of laboratory aggression. Rev. Gen. Psychol. 1, 19-41.

Berkowitz,L.(1989). Frustration-aggression hypothesis: examination and reformulation. Psychol. Bull. 106, 59-73.
Berkowitz, L. (1990). On the formation and regulation of anger and aggression. A cognitive-neoassociationistic analysis. Am. Psychol. 45, 494.

Berkowitz, L., and Harmon-Jones, E. (2004). Toward an understanding of the determinants of anger. Emotion 4, 107-130.

Buss, K. A., and Goldsmith, H. H. (1998). Fear and anger regulation in infancy: Effects on the temporal dynamics of affective expression. Child Development 69, 359-374.

\section{DISCUSSION}

The current study was designed to test the hypothesis that anger is associated with approach tendencies using face-valid full-body movements and measuring RTs. The main result is that anger was associated with a shift in approach-avoidance balance in favor of approach. Arousal and negative affect were ruled out as alternative explanations as a similar shift was not evident in participants who underwent an anxiety induction.

A possible limitation of the current study is that the anxiety induction was not as potent as the anger induction (as indicated by the mood checks). Nonetheless, the participants in this group reported increases in other negative feelings relative to baseline, yet did not show even a slight shift in their approach/avoidance RTs. Another possible limitation of our study might be that the participants in the anger group were asked to choose the noise level to administer and when doing so, they had to lean forward in order to type in their selection on the keyboard. Thus, these participants might have been primed to move forward during the competition phase, making their forward movements quicker. However, the results show that the tendency to approach within the anger group existed only in blocks 2 and 3 when they were angrier, and not in block 1 . This was so despite the fact that the requirement to choose the noise level existed in all blocks.

The final limitation of our study is that all the participants were female. Nonetheless, in the aforementioned pilot study, we did not exclude males from participating. This study included only an anger group and a control group, and revealed a significant triple interaction between group, block, and movement direction that reflected the same trend as that reported here, thus suggesting that the results are applicable to both sexes.

The present results provide clear and important support for the hypothesis that anger is associated with increased full-body approach/avoidance motor behavior. The hypothesis than anger is related to approach motivation has thus far been based on analog movements such as flexion and extension rather than movements involving the full body (e.g., Adams et al., 2006; Wilkowski and Meier, 2010). Our study is novel in that it offers a method which more closely resembles approach and avoidance behaviors outside the laboratory, as our participants actually regulated the distance to the stimulus by walking closer to it or away from it.

\section{ACKNOWLEDGMENTS}

This work was supported by a research grant to Nachshon Meiran from the Israel Science Foundation. We wish to thank American Journal Experts for English proofreading.

Carver, C. S. (2004). Negative affects deriving from the behavioral approach system. Emotion 4, 3-22.

Carver, C. S., and Harmon-Jones, E. (2009). Anger is an approach-related affect: evidence and implications. Psychol. Bull. 135, 183-204.

Centerbar, D. B., and Clore, G. L. (2006). Do approach-avoidance actions create attitudes? Psychol. Sci. 17, 22-29.

Chajut, E., Mama, Y., Levy, L., and Algom, D. (2010). Avoiding the approach trap: a response bias theory of the emotional Stroop effect. J. Exp. Psychol. Learn. Mem. Cogn. 36, 1567-1572.

Chen, M., and Bargh, J. A. (1999). Consequences of automatic evaluation: immediate behavioral predispositions to approach or avoid the stimulus. Pers. Soc. Psychol. Bull. 25, 215.

Coan, J. A., and Allen, J. J. B. (2004). Frontal EEG asymmetry as a moderator and mediator of emotion. Biol. Psychol. 67, 7-50. 
Cretenet, J., and Dru, V. (2004). The influence of unilateral and bilateral arm flexion versus extension on judgments: an exploratory case of motor congruence. Emotion 4, 282-294.

Frijda, N. H. (1986). The Emotions. Paris: Les Éditions de la Maison des sciences de l'homme.

Harmon-Jones, E. (2003). Clarifying the emotive functions of asymmetrical frontal cortical activity. Psychophysiology 40, 838-848.

Harmon-Jones, E. (2007). Trait anger predicts relative left frontal cortical activation to anger-inducing stimuli. Int. J. Psychophysiol. 66, 154-160.

Harmon-Jones, E., and Allen, J. J. B. (1998). Anger and frontal brain activity: EEG asymmetry consistent with approach motivation despite negative affective valence. J. Pers. Soc. Psychol. 74, 1310-1316.

Harmon-Jones, E., Lueck, L., Fearn, M., and Harmon-Jones, C. (2006). The effect of personal relevance and approach-related action expectation on relative left frontal cortical activity. Psychol. Sci. 17, 434-440.

Harmon-Jones, E., and Sigelman, J. (2001). State anger and prefrontal brain activity: evidence that insultrelated relative left-prefrontal activation is associated with experienced anger and aggression. J. Pers. Soc. Psychol. 80, 797-803.

Harmon-Jones, E., Vaughn-Scott, K., Mohr, S., Sigelman, J., and HarmonJones, C. (2004). The effect of manipulated sympathy and anger on left and right frontal cortical activity. Emotion 4, 95-101.
Hoeksma, J. B., Oosterlaan, J., Schipper, E., and Koot, H. (2007). Finding the attractor of anger: bridging the gap between dynamic concepts and empirical data. Emotion 7, 638-648.

Kassinove, H. (1995). Anger Disorders: Definition, Diagnosis, and Treatment. New York: Hemisphere Pub.

Keret, E. (2002). Anihu. Tel-Aviv: ZmoraBeitan [Hebrew].

Lavender, T., and Hommel, B. (2007). Affect and action: towards an eventcoding account. Cogn. Emot. 21, 1270-1296.

Lazarus, R. S. (1966). Psychological Stress and the Coping Process. New York: McGraw-Hill.

Lewis, M., Alessandri, S. M., and Sullivan, M.W. (1990).Violation of expectancy, loss of control, and anger expressions during extinction: antecedents and consequences. Infant Behav. Dev. 15, 443-452.

Lobbestael, J., Arntz, A., and Wiers, R. W. (2007). How to push someone's buttons: a comparison of four angerinduction methods. Cogn. Emot. 22, 353-373.

Markman,A. B., and Brendl, C. M. (2005). Constraining theories of embodied cognition. Psychol. Sci. 16, 6-10.

Marsh, A. A., Ambady, N., and Kleck, R. E. (2005). The effects of fear and anger facial expressions on approach-and avoidance-related behaviors. Emotion 1, 119-124.

Moritz, S., Veckenstedt, R., Randjbar, S., Hottenrott, B., Woodward, T. S., Eckstaedt, F. V., Schmidt, C., Jelinek, L., and Lincoln, T.M. (2009). Decision making under uncertainty and mood induction: further evidence for liberal acceptance in schizophrenia. Psychol. Med. 39, 1821-1829.

Neumann, R., and Strack, F. (2000). Approach and avoidance: the influence of proprioceptive and exteroceptive cues on encoding of affective information. J. Pers. Soc. Psychol. 79 , 39-48.

Newhagen, J. E. (1998). TV news images that induce anger, fear, and disgust: effects on approach-avoidance and memory. J. Broadcast. Electron. Media 42, 265-276.

Parrott, W. (2001). Implications of dysfunctional emotions for understanding how emotions function. Rev. Gen. Psychol. 5, 180-186.

Quartana, P. J., and Burns, J. W. (2007) Painful consequences of anger suppression. Emotion 7, 400-414.

Rusting, C. L., and Nolen-Hoeksema, S. (1998). Regulating responses to anger: effects of rumination and distraction on angry mood. J. Pers. Soc. Psychol. 74, 790-803.

Solarz, A. K. (1960). Latency of instrumental responses as a function of compatibility with the meaning of eliciting verbal signs. J. Exp. Psychol. 59, 239-245.

Spielberger, C. D. (1996). State-Trai Anger Expression Inventory: STAXI Professional Manual. Tampa, FL: Psychological Assessment Resources.

Tamir, M., Mitchell, C., and Gross, J. J. (2008). Hedonic and instrumental motives in anger regulation. Psychol. Sci. 19, 324-328.

van Dantzig, S., Pecher, D., and Zwaan, R. A. (2008). Approach and avoidance as action effects. Q. J. Exp. Psychol. 61, 1298-1306.

Weiss, H.M., Suckow, K., and Cropanzano, R. (1999). Effects of justice conditions on discrete emotions. J. Appl. Psychol. 84, 786-794.

Wentura, D., Rothermund, K., and Bak, P. (2000). Automatic vigilance: the attention-grabbing power of approach-and avoidance-related social information. J. Pers. Soc. Psychol. 78, 1024-1037.

Wilkowski, B. M., and Meier, B. P. (2010). Bring it on: angry facial expressions potentiate approach-motivated motor behavior. J. Pers. Soc. Psychol. 98, 201-210.

Conflict of Interest Statement: The authors declare that the research was conducted in the absence of any commercial or financial relationships that could be construed as a potential conflict of interest.

Received: 20 July 2010; accepted: 04 February 2011; published online: 15 February 2011.

Citation: Mayan I and Meiran N (2011) Anger and the speed of full-body approach and avoidance reactions. Front. Psychology 2:22. doi: 10.3389/fpsyg.2011.00022

This article was submitted to Frontiers in Emotion Science, a specialty of Frontiers in Psychology.

Copyright (c) 2011 Mayan and Meiran. This is an open-access article subject to an exclusive license agreement between the authors and Frontiers Media SA, which permits unrestricted use, distribution, and reproduction in any medium, provided the original authors and source are credited. 